\title{
Event-related electroencephalographic responses in children at familial high risk for schizophrenia or bipolar disorder: a single-site EEG sub-study of the Danish High Risk and Resilience Study - VIA 11
}

Anna Hester Ver Loren van Themaat ${ }^{1,2 *}$, Kit Melissa Larsen ${ }^{1,2}$, Bob Oranje ${ }^{3,4}$, Leo Tomasevic ${ }^{2}$, Line Korsgaard Johnsen ${ }^{1,2}$, William Frans Christiaan Baaré ${ }^{2}$, Ayna Baladi Nejad ${ }^{2}$, Heike Eichele ${ }^{5}$, Anne Amalie Elgaard Thorup ${ }^{6,7,8}$, Merete Nordentoft ${ }^{6,7,8}$, Kerstin Jessica Plessen ${ }^{1,8,9}$, Hartwig Roman Siebner ${ }^{2,6,10^{*}}$

${ }^{1}$ Child and Adolescent Mental Health Centre, Mental Health Services, Capital Region of Denmark, Copenhagen, Denmark; ${ }^{2}$ Danish Research Centre for Magnetic Resonance, Centre for Functional and Diagnostic Imaging and Research, Copenhagen University Hospital Hvidovre, Denmark; ${ }^{3}$ Center for Clinical Intervention and Neuropsychiatric Schizophrenia Research and Center for Neuropsychiatric Schizophrenia Research (CNSR), Mental Health Services, Capital Region of Denmark, Copenhagen, Denmark; ${ }^{4}$ Department of Psychiatry, UMC Utrecht Brain Center, Utrecht, The Netherlands; ${ }^{5}$ Department of Biological and Medical Psychology, University of Bergen, Bergen, Norway; ${ }^{6}$ Department of Clinical Medicine, Faculty of Health and Medical Sciences, University of Copenhagen, Copenhagen, Denmark; ${ }^{7}$ Mental Health Centre Copenhagen, Mental Health Services, Capital Region of Denmark, Copenhagen, Denmark; ${ }^{8}$ The Lundbeck Foundation Initiative for Integrative Psychiatric Research (iPSYCH), Aarhus, Denmark; ${ }^{9}$ Division of Child and Adolescent Psychiatry, Department of Psychiatry, Lausanne, University Hospital, Lausanne, Switzerland; ${ }^{10}$ Department of Neurology, Copenhagen University Hospital Bispebjerg, Copenhagen, Denmark.

*Corresponding authors

Email: anna.hester.ver.loren.van.themaat.01@regionh.dk

Email: hartwig.roman.siebner@regionh.dk 


\begin{abstract}
Aim: Studying children of parents with schizophrenia or bipolar disorder, i.e. who are at familial high-risk of developing similar disorders, offers the possibility to identify abnormalities preceding the emergence of mental health disorders during adolescence. This paper reports the rationale and methodology of a single-site electroencephalography-study of the register-based Danish High Risk and Resilience Study-VIA 11. Focusing on event-related electroencephalographic read-outs that have previously been proposed as endophenotypes for schizophrenia, we set out to retrieve these endophenotypes in children at familial high-risk.

Method: Event-related cortical responses to repetitive auditory stimuli or visuospatial flanker stimuli will be recorded with 128-channel electroencephalography in eleven-year-old children with one or two parents diagnosed with schizophrenia spectrum psychosis $(n>40)$ or bipolar disorder $(n>40)$ and control children without familial high-risk $(n>40)$. We will test for between-group differences in auditory processing, focusing on the auditory steady-state response and mismatch negativity. We will also assess between-group differences in visually evoked cortical activity implicated in the resolution of a visuomotor response conflict such as P3b potential and lateralized readiness potential. We will further examine whether the individual expression of these electroencephalographic read-outs scale with clinical characteristics.

Conclusions: The study will clarify whether potential electroencephalographic-derived endophenotypes are expressed in children of parents with schizophrenia and possibly also in children of parents with bipolar disorder. The multiple electrophysiological-based read-outs of brain reactivity will enable additional exploratory analyses. Together, the study will contribute to current attempts to validate and identify electroencephalographic-based endophenotypes of vulnerability for mental health disorders.
\end{abstract}

Keywords: Bipolar disorder, electroencephalography, endophenotypes, familial high-risk, offspring, schizophrenia. 


\section{Introduction}

Schizophrenia (SZ) and bipolar disorder (BP) are among the most severe mental disorders imposing a huge impact on patients and their families (Millier et al., 2014) and show considerable overlap in their genetic, symptomatic and neurobiological characteristics (Purcell et al., 2009). Behavioural, cognitive and emotional abnormalities are present before the prodromal phases of both illnesses, though deficits are more severe in those who will develop SZ (Bora, 2016), which argues for a stronger neurodevelopmental nature in SZ compared to BP (Owen, O'Donovan, Thapar, \& Craddock, 2011). Studies that include children born to parents with SZ or BP, which are at a familial high risk (FHR) for developing these disorders, provide the opportunity to investigate vulnerability and protective factors, as well as factors of resilience in those who present with the typical endophenotypes, but do not develop the disorder (Rasic, Hajek, Alda, \& Uher, 2014).

Prospective longitudinal FHR-studies suggested several cognitive endophenotypes for both disorders (Bora \& Ozerdem, 2017; Hameed \& Lewis, 2016). Few studies included electroencephalography (EEG), a non-invasive and cost-effective technique to investigate early information-processing abilities (Friedman \& Squires-Wheeler, 1994; Schreiber, Stolz, Rothmeier, Kornhuber, \& Born, 1989). EEG provides a direct measure of electrical activity with high temporal resolution, which is essential to study oscillatory activity and event-related potentials (ERPs). Furthermore, EEG has greatly contributed to the search for endophenotypes in psychiatry; in SZ (Earls, Curran, \& Mittal, 2016), BP (Ethridge et al., 2015) and in other psychiatric disorders (Riva et al., 2018). The following section describes three aspects of neural mechanisms being tested as part of the current study 1) auditory steady-state responses, 2) automatic cortical processing of changes in regularity of auditory input and 3) event-related cortical responses of visuomotor control.

\section{Auditory steady-state responses}

Auditory steady-state responses (ASSRs) are evoked by brief tones or clicks and provide readily available, noninvasive means of probing impaired neural gamma synchrony in the auditory system (Larsen et al., 2017; Thune, Recasens, \& Uhlhaas, 2016). The ability to generate $40 \mathrm{~Hz}$ ASSR relies on the GABAergic interneurons (Bartos, Vida, \& Jonas, 2007; Sohal, Zhang, Yizhar, \& Deisseroth, 2009; Traub et al., 2003) by exerting a finely timed inhibition onto the pyramidal cells. ASSR at 40 $\mathrm{Hz}$ is well-studied in patients with chronic SZ; 17 out of 20 studies reported reductions in power and phase-locking of $40 \mathrm{~Hz}$ ASSR compared to healthy subjects (Thune et al., 2016). Additionally, 
unaffected first-degree relatives (UFDR) of patients with SZ showed reduced $40 \mathrm{~Hz}$ ASSR (Rass et al., 2012). The few studies that investigated ASSR in patients with BP reported similar results (O'Donnell, Hetrick, et al., 2004; Rass et al., 2010) suggesting circuit dysfunctions in both disorders. Furthermore, evidence shows that deficits in early gamma-band responses arise after the onset of psychosis while the late gamma-band response is already premorbid impaired (Tada et al., 2016). These findings indicate its potential role as endophenotype for SZ whereas their potential role as an endophenotype of BP is not well elucidated.

Automatic cortical processing of changes in regularity of auditory input

The preconscious process of detecting change in regularity of auditory input is expressed by the mismatch negativity (MMN). MMN reflects an automatic predictive processing system that is evoked when an expectation regarding upcoming stimuli is violated. This results in a fronto-temporal negative deflection that peaks between 150-250 ms after the mismatching stimulus (Näätänen, 1990). MMN amplitude reductions are consistently found in patients with chronic SZ, with effect sizes (ES) between 0.95 and 0.99 and are greatest in response to duration-deviant tones (Earls et al., 2016; Umbricht \& Krljes, 2005) and are also observed in UFDR of patients with SZ (Earls et al., 2016). MMN alterations are associated with poorer psychosocial functioning and ratings on global functioning in daily life (Light, Swerdlow, \& Braff, 2007) and are also reported in children with autism (Vlaskamp et al., 2017) and adolescents with ADHD (Rydkjaer et al., 2017). Recent findings revealed that MMN amplitude reductions may be due to a decreased connectivity from higher to lower cortical areas, indicative of predictive processes going wrong (Larsen et al., 2018). Evidence of MMN changes in patients with BP is more controversial and meta-analyses reported moderate ESs between 0.37 and 0.45 (Chitty, Lagopoulos, Lee, Hickie, \& Hermens, 2013; Erickson, Ruffle, \& Gold, 2016). UFDR of patients with BP showed no MMN deficits compared to healthy subjects (Hall et al., 2009; Umbricht et al., 2003). These findings suggest MMN deficits as potential endophenotype for SZ, whereas this has not yet been documented for BP.

Event-related cortical responses of visuomotor control

In visuomotor tasks assessing interference control, e.g. the flanker task (Eriksen, 1995), cortical responses reflect higher cognitive processes. To investigate neural correlates of interference control, three ERP-components N200, P3b and the lateralized readiness potential (LRP) are of interest (Coles, 1989; Eichele et al., 2016; Xie, Ren, Cao, \& Li, 2017). N200 is a frontocentral negative peak at 
approximately $200 \mathrm{~ms}$ after stimulus onset and its amplitude is enhanced in tasks eliciting response conflict (Heil, 2000; Kopp, Rist, \& Mattler, 1996). P3b is a parietal positive peak at approximately $300 \mathrm{~ms}$ after stimulus onset. P3b amplitude reflects the degree of cognitive demand during task processing and P3b latency reflects the time required to detect a target stimulus (Polich, 2007). Alterations in N200 and P3b amplitudes reflect impaired attention-dependent processing in higher cortical regions and are linked to other disorders with attention and inhibition disturbances, such as ADHD (Tsai, Hung, \& Lu, 2012). Specifically, reductions in N200 amplitudes have been shown in patients with SZ, but not in patients with BP (O'Donnell, Vohs, Hetrick, Carroll, \& Shekhar, 2004), whereas both patient groups show reduced P300 amplitudes and prolonged P300 latencies (Jahshan et al., 2012; O'Donnell, Vohs, et al., 2004), which also precede psychosis onset (Hamilton et al., 2019). UFDR of patients with SZ, compared to healthy subjects, display prolonged N200 latencies (Frangou et al., 1997; Schreiber et al., 1989), reduced P300 amplitudes (ES: 0.52-0.61) and prolonged P300 latencies (ES: 0.44-0.50) (Bramon et al., 2005; Earls et al., 2016). UFDR of patients with BP only show smaller P300 amplitudes (Hall et al., 2009; Schulze et al., 2008), though fewer studies have been conducted. Finally, the LRP reflects the difference in motor cortex activation between the two cerebral hemispheres and is related to response preparation and execution (Coles, 1989). Delayed onset of the LRP, i.e. lengthening of motor response selection and preparation processes, are present in both SZ and BP (Van Voorhis et al., 2019). When our cohort was aged 7, children with FHR-SZ showed motor impairments relative to control children (Burton et al., 2017), which gives reason to study the LRP. In addition to the stimulus-locked ERPs, response-locked ERPs such as the errorrelated negativity (ERN) and error positivity (Pe) allow us to study processes of error monitoring. Specifically, the ERN indexes automatic error detection and arises immediately after an erroneous response (Ford, Jorgensen, Roach, \& Mathalon, 2009). Subsequently, Pe emerges approximately 300 ms after an erroneous response and reflects the evaluation and awareness of the incorrect response (Davies, Segalowitz, \& Gavin, 2004).

We designed a study to identify potential electrophysiological endophenotypes for SZ and/or BP. Our primary aim is to clarify whether suggested electrophysiological-based endophenotypes can be retrieved in this FHR-cohort. Therefore, EEG will be recorded while children undergo a gamma entrainment paradigm to measure ASSR, an auditory oddball paradigm to measure MMN and a modified flanker task to measure the ERP-components N200, P3b and LRP. We hypothesize that children at FHR-SZ relative to control children show (1) reductions in power and inter-trial phase 
coherence of the ASSR in response to regular auditory stimulation at $40 \mathrm{~Hz}$, (2) reduced amplitudes of the MMN response, (3) altered N200, P3b and LRP in more attentional demanding conditions in the flanker task that require suppression of a prepotent response, and (4) altered ERN and Pe after the commission of an error. Given the overlap in SZ and BP, we expect that EEG outcome measures of children at FHR-BP will lie between those of FHR-SZ and control children. Further, we will perform exploratory analyses to identify novel endophenotypes related to GABAergic circuitry dysfunction through computational modelling (Shaw et al., 2019; Symmonds et al., 2018) within the network generating the ASSR as well as altered connectivity in the MMN network. Additionally, we expect associations between the individual EEG-outcomes and severity of behavioural problems, especially with symptomatology of autism and ADHD. Finally, we propose a combination of altered functional patterns constituting complementary network biotypes conferring risk for psychosis. This opens up for follow-up studies to investigate resilience factors.

\section{Methods}

\section{Study design}

This study was approved by The Danish Data Protection Agency as part of the Danish High Risk and Resilience Study - VIA 11 (Thorup et al., 2018). The Danish High Risk and Resilience Study - VIA 7 was initiated in 2013 when children were 7 years of age (Thorup et al., 2015) and a collaboration of two research sites in Denmark (Copenhagen and Aarhus). The EEG sub-study was added to the first follow-up (VIA 11) but only performed at the research site in Copenhagen, at the Danish Research Centre for Magnetic Resonance (DRCMR), Copenhagen University Hospital Hvidovre. Children of the cohort were evenly distributed over the two research sites based on place of residence, FHR status, and gender. EEG was performed by trained researchers who are blinded to the group status of the parent. Parents or legal guardians of participating children complete a written informed consent after written and oral explanation of the study procedures. Children receive a verbal, childfriendly explanation of the study. Although the current EEG study is cross-sectional, we plan to incorporate EEG in future waves of the Danish High Risk and Resilience Study that are intended to occur every four years.

\section{Participants}

This study will recruit at least 40 children at FHR-SZ, 40 children at FHR-BP and 40 control children. Eleven-year-old children with either zero, one, or two parent(s) diagnosed with schizophrenia 
spectrum psychosis (defined as SZ, delusional disorder and schizoaffective disorder; ICD 10-codes: F20, F22 and F25 or ICD 8-codes: 295, 297, 298.29, 289.39, 298.89, 298.99) or BP (ICD 10-code F30 and F31 or ICD 8-codes: 296.19, 296.39) were recruited from VIA 7 (Thorup et al., 2015) and testing commenced in May 2017. The original cohort of VIA 7 was recruited through the Danish Civil Registration System and the Danish Psychiatric Central Research register (Mors, Perto, \& Mortensen, 2011) and was required to have Danish as native language. Children not at FHR (population-based controls; PBC) were matched to children at FHR-SZ on municipality, sex and age. Children at FHR-BP were comparable to the other groups in terms of age and gender. Power calculations for the EEG read-outs were difficult to make due to a low number (or lack) of previous studies including children with FHR-SZ and FHR-BP. Our sample size of at least 40 children per group (total $\mathrm{N}=120$ ) is high compared to the existing studies (Friedman, Cornblatt, Vaughan, \& Erlenmeyer-Kimling, 1988; Schreiber, Stolz-Born, Kornhuber, \& Born, 1992) and compared to studies including young and adult individuals with CHR for psychosis (Bruggemann, Stockill, Lenroot, \& Laurens, 2013; Lepock et al., 2018), although effect sizes may be different due to different clinical characteristics.

\section{Procedure}

After generally explaining EEG, participants are seated in a comfortable chair in a quiet room. Participants are carefully instructed about the experimental procedures. Three experimental tasks are presented in the following fixed-order; gamma entrainment paradigm, (2×6 minutes); modified flanker task (Eichele et al., 2016; Eriksen, 1995), (2×6 minutes); and an auditory oddball paradigm (MMN) (Düring, Glenthøj, \& Oranje, 2016), (16 minutes). Training of the modified flanker task takes place prior to EEG testing, at an earlier MRI session in which the same task is also assessed (Thorup et al., 2018). In case children do not want to be scanned, the flanker-training is performed before the EEG session. The experimental part of the EEG takes approximately 45 minutes (including breaks).

\section{Electroencephalography}

EEG data is recorded with a Biosemi ActiveTwo (BioSemi, Amsterdam, Netherlands) 128-channel system at a sampling frequency of $4096 \mathrm{~Hz}$. External electrodes record electro-oculography. Auditory stimuli are delivered binaurally via insert-earphones (E-ARTONE 3A). EEG signals will be preprocessed and analyzed using the FieldTrip toolbox (Oostenveld, Fries, Maris, \& Schoffelen, 2011), 
SPM, Brain Electrical Source Analysis (MEGIS Software GmbH, Gräfelfing, Germany) and customized scripts.

\section{Paradigms/tasks}

1) ASSR: participants are presented with auditory stimuli containing sequences of $40 \mathrm{~Hz}$ click trains. One sequence' stimulation lasts one second, followed by a two-second pause. We use a regular and irregular condition, see Larsen et al. (2017). In the regular condition, clicks with the duration of one second are distributed regularly at a rate of 40 clicks per second, while clicks are distributed irregularly over the one-second stimulation period in the irregular (control) condition. Each condition is recorded in separate blocks of six minutes. Participants are asked to relax while fixating on a fixation cross (see Figure 1).

2) MMN: participants are presented with an auditory oddball paradigm (Düring et al., 2016). This paradigm consists of 1800 stimuli in total with standard tones (76\% occurrence) and deviant tones (8\% occurrence) of three kinds. The standard tone is a pure tone with a frequency of $1000 \mathrm{~Hz}$, an intensity of $75 \mathrm{~dB}$ and a duration of $50 \mathrm{~ms}$. Deviant tones differ either in frequency $(1200 \mathrm{~Hz}, 75 \mathrm{~dB}$, $50 \mathrm{~ms}$ ), duration $(1000 \mathrm{~Hz}, 75 \mathrm{~dB}, 100 \mathrm{~ms})$ or its combination $(1200 \mathrm{~Hz}, 75 \mathrm{~dB}, 100 \mathrm{~ms})$. Participants are asked to relax while watching a muted movie.

3) Modified flanker task: participants perform a modified flanker task (Eichele et al., 2016; Eriksen, 1995) running in E-Prime 2 (Psychology Software Tools, 2012). Trials start with a 700 ms fixation dot at the center of the screen. Six flanking arrows appear for $100 \mathrm{~ms}$ followed by the target arrow, which is present for $1000 \mathrm{~ms}$ in the middle of the six flankers. Flanking and target arrows point in the same (congruent condition, 50\%) or in the opposite direction (incongruent condition, 50\%). Participants are instructed to press the left button with their left thumb when the target arrow points to the left and vice versa. Four-hundred trials are presented in two blocks in fixed-order between subjects each lasting six minutes. An exclamation mark appears when participants respond too slow, either because of exceeding the response window (1000 ms) or their personal threshold calculated during the training, i.e. mean reaction time $(\mathrm{RT})+1.5 \mathrm{x}$ SD of the mean RT on 10 incongruent trials.

\section{Functional and clinical measures}

Children will be assessed with the Reynolds Intellectual Screening Test (RIST) (Reynolds, Kamphaus, \& Lutz, 2003), for estimating IQ. The Schedule for Affective Disorders and 
Schizophrenia for School-Age Children - Present and Lifetime Version (K-SADS) (Kaufman et al., 1997) will be used to ascertain presence of psychiatric disorders and subsyndromal symptomatology. We included the following measures to assess daily functioning, global problem behaviour, positive symptomatology and specific severity of autism and ADHD. The Children's Global Assessment Scale (CGAS) (Shaffer et al., 1983) rates a child's psychological, social and daily functioning. The Childhood Behavior Checklist (CBCL) (Bilenberg, 1999) reports on various behaviours and emotions in children. Psychotic-Like Experiences (PLE) (Miller et al., 2003) assesses sub-threshold psychoticlike experiences. The Social Responsiveness Scale (SRS) (Constantino \& Gruber, 2005) provides an index of the severity of autistic traits. The Attention-Deficit/Hyperactivity Disorder - Rating Scale (ADHD-RS) (Makransky \& Bilenberg, 2014) assesses the severity of ADHD-symptomatology.

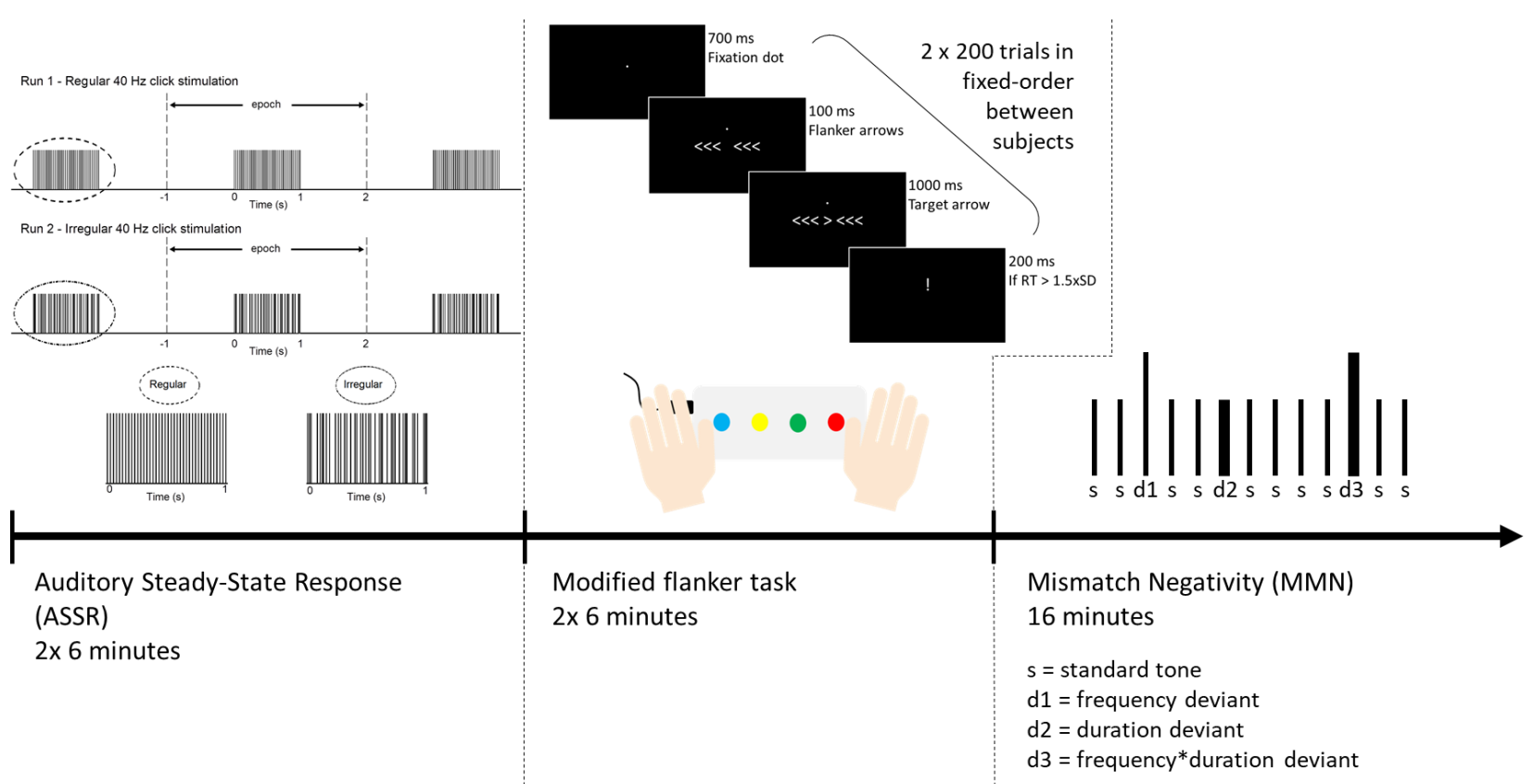

Figure 1. The paradigms presented during EEG recordings. From left to right, are the gamma entrainment paradigm (Larsen et al., 2017) to study the auditory steady-state response (ASSR), the modified flanker task (Eichele et al., 2016; Eriksen, 1995) to elicit the N200, P3b and LRP, and an auditory oddball paradigm (Düring et al., 2016) to elicit mismatch negativity (MMN). The ASSR image is adapted with permission from (Larsen et al., 2017).

\section{Statistical analyses}

Demographic and clinical characteristics will be analyzed by using parametric (one-way analysis of variance (ANOVA)) or non-parametric methods where appropriate. Behavioural performance on the flanker task will be evaluated following the statistical analyses in Ridderinkhof (2002). To assess group differences in the EEG outcome variables we will employ two different approaches; one 
hypothesis-driven approach assessing differences in prespecified channels known to produce highest responses for the given task (Handy, 2005) as well as a more unbiased assumption-free approach. For the primary aim, we will test for group differences on the EEG outcome variables from the ASSR, MMN and flanker task by using a repeated-measures analysis of (co)variance (RM-AN(C)OVA) with the EEG outcome variable as the dependent variable, group-membership as between-subject factor (children at FHR-SZ, children at FHR-BP and PBC children) and condition as within-subject factor (i.e. congruent versus incongruent condition or correct versus incorrect for the flanker task). MMN is considered to show the highest amplitude at the frontal/midline EEG-channels (Hall et al., 2007; Light et al., 2007), whereas highest N200 and P3b amplitudes are typically found at frontal/midline and centro/parietal electrodes (Xie et al., 2017). Electrodes C3 and C4 will be used for the calculation of the LRP (Coles, 1989). ERN and Pe are considered maximal at frontal and central electrodes (Davies et al., 2004). Bonferroni correction for multiple comparisons will be applied to post hoc tests.

For the secondary aim, we will search for differences over the entire electrode-time (or frequency) volume and use random field theory to correct for multiple comparisons (Kilner \& Friston, 2010). Using this approach, we can capture if differences are present outside of the expected component while still controlling for multiple comparisons. For the ASSR data we will use Dynamic Causal modelling (DCM) with the canonical microcircuit model to tap into potential GABAergic circuitry dysfunction (Shaw et al., 2019; Symmonds et al., 2018). For the MMN task we will employ DCM for ERPs (David et al., 2006) to infer on group differences in the connectivity of the underlying network generating the MMN responses. For each of the EEG outcome variables, we will explore how these are related to the clinical variables using either parametric or non-parametric correlation methods. Finally, we will use a multivariate machine learning approach to merge all parameters to learn from the data by doing a cluster analysis to look at potential overlaps.

Age and sex will be included in all analyses if they covary significantly. We will conduct a post hoc test for the effect of IQ. P-values will be reported significant if below 0.05 .

\section{Discussion}

This paper describes the rationale and methodology of an EEG-investigation of 11-year-old children at FHR-SZ and FHR-BP and PBC children. Evidence suggests a considerable overlap in genetic, neuropsychological and neurobiological characteristics between SZ and BP (Purcell et al., 2009). However, the search for endophenotypes that could identify those individuals at highest risk to develop these disorders remains ongoing. This study is the first EEG-investigation in a young, large 
FHR-sample of the same age assessing several electrophysiological measures within the context of a longitudinal cohort-study, namely ASSR, MMN, N200, P3b, LRP, ERN and Pe. By doing so, we aim to 1) identify potential electrophysiological endophenotypes for SZ and/or BP, 2) explore to what extent clinical characteristics correlate with these electrophysiological measures and 3) identify electrophysiological profiles that may distinguish children at FHR-SZ from children at FHR-BP, which can be validated in future follow-up studies.

We expect that our findings will contribute to gaining a deeper insight into potential electrophysiological endophenotypes for SZ and BP. Our findings may pave the way to follow-up studies on resilience factors because not all vulnerable children will develop the disorders. Finally, a better understanding of the underlying risk markers of SZ and BP may not only contribute to early diagnostic precision but also to the development of interventions designed to prevent or mitigate the individual expression of these severely disabling disorders. 


\section{Acknowledgments}

We are grateful to the participants and families who invested their time and energy in order to participate in our study. We would also like to express our gratitude to the staff involved in the Danish High Risk and Resilience Study VIA 7 in collecting the cohort in 2013, their advice, support and coordination to successfully start and continue in VIA 11. The staff involves Birgitte Klee Burton, Ditte Vestbjerg Ellersgaard, Camilla Jerlang Christiani, Nicoline Hemager, Katrine Søborg Spang, Ditte Lou Gantriis, Aja Neergaard Greve, Mette Skjærbæk, Anne Ranning, Kate Kold Zahle, Line Carmichael and Jessica Ohland. We highly appreciate the efforts of Benthe Emke Vink, Daban Sulaiman and Jonas Schultz Ingerslev for their dedicated assistance during data acquisition.

\section{Conflict of interest statement}

HRS has received honoraria as speaker from Sanofi Genzyme, Denmark and Novartis, Denmark, as consultant from Sanofi Genzyme, Denmark and as senior editor (NeuroImage) from Elsevier Publishers, Amsterdam, The Netherlands. He has received royalties as book editor from Springer Publishers, Stuttgart, Germany. The other authors declare they have no competing interests. 


\section{References}

Bartos, M., Vida, I., \& Jonas, P. (2007). Synaptic mechanisms of synchronized gamma oscillations in inhibitory interneuron networks. Nat Rev Neurosci, 8(1), 45-56. doi:10.1038/nrn2044

Bilenberg, N. (1999). The Child Behavior Checklist (CBCL) and related material: standardization and validation in Danish population based and clinically based samples. Acta Psychiatric Scandinavia, 100, 2-52.

Bora, E. (2016). Differences in cognitive impairment between schizophrenia and bipolar disorder: Considering the role of heterogeneity. Psychiatry Clin Neurosci, 70(10), 424-433. doi:10.1111/pen. 12410

Bora, E., \& Ozerdem, A. (2017). A meta-analysis of neurocognition in youth with familial high risk for bipolar disorder. Eur Psychiatry, 44, 17-23. doi:10.1016/j.eurpsy.2017.02.483

Bramon, E., McDonald, C., Croft, R. J., Landau, S., Filbey, F., Gruzelier, J. H., . . Murray, R. M. (2005). Is the P300 wave an endophenotype for schizophrenia? A meta-analysis and a family study. Neuroimage, 27(4), 960-968. doi:10.1016/j.neuroimage.2005.05.022

Bruggemann, J. M., Stockill, H. V., Lenroot, R. K., \& Laurens, K. R. (2013). Mismatch negativity $(\mathrm{MMN})$ and sensory auditory processing in children aged 9-12 years presenting with putative antecedents of schizophrenia. Int J Psychophysiol, 89(3), 374-380. doi:10.1016/j.ijpsycho.2013.05.008

Burton, B. K., Thorup, A. A., Jepsen, J. R., Poulsen, G., Ellersgaard, D. V., Spang, K. S., . . . Plessen, K. J. (2017). Impairments of motor function among children with a familial risk of schizophrenia or bipolar disorder at 7 years old in Denmark: an observational cohort study. The Lancet Psychiatry, 4(5), 400-408. doi:10.1016/s2215-0366(17)30103-7

Chitty, K. M., Lagopoulos, J., Lee, R. S., Hickie, I. B., \& Hermens, D. F. (2013). A systematic review and meta-analysis of proton magnetic resonance spectroscopy and mismatch negativity in bipolar disorder. Eur Neuropsychopharmacol, 23(11), 1348-1363. doi:10.1016/j.euroneuro.2013.07.007

Coles, M. G. H. (1989). Modern Mind-Brain Reading: Psychophysiology, Physiology, and Cognition. Psychophysiology, 26(3), 251-269.

Constantino, J. N., \& Gruber, C. P. (2005). Social responsive scale (SRS) manual. Los Angeles, CA: Western Psychological Services.

David, O., Kiebel, S. J., Harrison, L. M., Mattout, J., Kilner, J. M., \& Friston, K. J. (2006). Dynamic causal modeling of evoked responses in EEG and MEG. Neuroimage, 30(4), 1255 1272. doi:10.1016/j.neuroimage.2005.10.045

Davies, P., Segalowitz, S. J., \& Gavin, W. J. (2004). Development of Response-Monitoring ERPs in 7- to 26-Year-Old. Developmental Neuropsychology, 25(3), 355-376. 
Düring, S., Glenthøj, B. Y., \& Oranje, B. (2016). Effects of Blocking D2/D3 Receptors on Mismatch Negativity and P3a Amplitude of Initially Antipsychotic Naïve, First Episode Schizophrenia Patients. International Journal of Neuropsychopharmacology, 19(3). doi:10.1093/ijnp/pyv109

Earls, H. A., Curran, T., \& Mittal, V. (2016). A Meta-analytic Review of Auditory Event-Related Potential Components as Endophenotypes for Schizophrenia: Perspectives From FirstDegree Relatives. Schizophr Bull, 42(6), 1504-1516. doi:10.1093/schbul/sbw047

Eichele, H., Eichele, T., Bjelland, I., Hovik, M. F., Sorensen, L., van Wageningen, H., . . Plessen, K. J. (2016). Performance Monitoring in Medication-Naive Children with Tourette Syndrome. Front Neurosci, 10, 50. doi:10.3389/fnins.2016.00050

Erickson, M. A., Ruffle, A., \& Gold, J. M. (2016). A Meta-Analysis of Mismatch Negativity in Schizophrenia: From Clinical Risk to Disease Specificity and Progression. Biol Psychiatry, 79(12), 980-987. doi:10.1016/j.biopsych.2015.08.025

Eriksen, C. W. (1995). The flankers task and response competition. a useful tool for investigating a variety of cognitive problems. Visual Cognition, 2, 101-118.

Ethridge, L. E., Hamm, J. P., Pearlson, G. D., Tamminga, C. A., Sweeney, J. A., Keshavan, M. S., $\&$ Clementz, B. A. (2015). Event-related potential and time-frequency endophenotypes for schizophrenia and psychotic bipolar disorder. Biol Psychiatry, 77(2), 127-136. doi:10.1016/j.biopsych.2014.03.032

Ford, J. M., Jorgensen, K. W., Roach, B. J., \& Mathalon, D. H. (2009). Error detection failures in schizophrenia: ERPs and FMRI. Int J Psychophysiol, 73(2), 109-117. doi:10.1016/j.ijpsycho.2009.02.005

Frangou, S., Sharma, T., Alarcon, G., Sigmudsson, T., Takei, N., Binnie, C., \& Murray, R. M. (1997). The Maudsley Family Study, II: Endogenous event-related potentials in familial schizophrenia. Schizophr Res, 23(1), 45-53. doi:10.1016/S0920-9964(96)00089-8

Friedman, D., Cornblatt, B., Vaughan, H., \& Erlenmeyer-Kimling, L. (1988). Auditory eventrelated potentials in children at risk for schizophrenia: the complete initial sample. Psychiatry Research, 26(2), 203-221

Friedman, D., \& Squires-Wheeler, E. (1994). Event-related potentials (ERPs) as indicators of risk for schizophrenia. Schizophr Bull, 20(1), 63.

Hall, M. H., Rijsdijk, F., Kalidindi, S., Schulze, K., Kravariti, E., Kane, F., . . Murray, R. M. (2007). Genetic overlap between bipolar illness and event-related potentials. Psychol Med, 37(5), 667-678. doi:10.1017/S003329170600972X

Hall, M. H., Schulze, K., Rijsdijk, F., Kalidindi, S., McDonald, C., Bramon, E., . . Sham, P. (2009). Are auditory P300 and duration MMN heritable and putative endophenotypes of psychotic bipolar disorder? A Maudsley Bipolar Twin and Family Study. Psychological Med, 39(8), 1277-1287. doi:10.1017/S0033291709005261 
Hameed, M. A., \& Lewis, A. J. (2016). Offspring of Parents with Schizophrenia: A Systematic Review of Developmental Features Across Childhood. Harv Rev Psychiatry, 24(2), 104117. doi:10.1097/HRP.0000000000000076

Hamilton, H. K., Roach, B. J., Bachman, P. M., Belger, A., Carrion, R. E., Duncan, E., . . Mathalon, D. H. (2019). Association Between P300 Responses to Auditory Oddball Stimuli and Clinical Outcomes in the Psychosis Risk Syndrome. JAMA Psychiatry. doi:10.1001/jamapsychiatry.2019.2135

Handy, T. C. (2005). Event-related potentials: A methods handbook: MIT press.

Heil, M., Osman, A., Wiegelmann, J., Rolke, B., \& Hennighausen, E. (2000). N200 in the EriksenTask: Inhibitory Executive Processes? Journal of Psychophysiology, 14, 218-225.

Jahshan, C., Wynn, J. K., Mathis, K. I., Altshuler, L. L., Glahn, D. C., \& Green, M. F. (2012). Cross-diagnostic comparison of duration mismatch negativity and P3a in bipolar disorder and schizophrenia. Bipolar Disord, 14(3), 239-248. doi:10.1111/j.1399-5618.2012.01008.x

Kaufman, J., Birmaher, B., Brent, D., Rao, U., Flynn, C., Moreci, P., . . Ryan, N. (1997). Schedule for affective disorders and schizophrenia for school-age children-present and lifetime version (K-SADS-PL): initial reliability and validity data. JOURNAL OF THE AMERICAN ACADEMY OF CHILD \& ADOLESCENT PSYCHIATRY, 36(7), 980-988.

Kilner, J. M., \& Friston, K. J. (2010). Topological inference for EEG and MEG. The Annals of Applied Statistics, 4(3), 1272-1290. doi:10.1214/10-aoas337

Kopp, B., Rist, F., \& Mattler, U. (1996). N200 in the flanker task as a neurobehavioral tool for investigating executive control. Psychophysiology, 33(3), 282-294.

Larsen, K. M., Morup, M., Birknow, M. R., Fischer, E., Hulme, O., Vangkilde, A., . . Garrido, M. I. (2018). Altered auditory processing and effective connectivity in 22q11.2 deletion syndrome. Schizophr Res. doi:10.1016/j.schres.2018.01.026

Larsen, K. M., Pellegrino, G., Birknow, M. R., Kjaer, T. N., Baare, W. F. C., Didriksen, M., . . Siebner, H. R. (2017). 22q11.2 Deletion Syndrome Is Associated With Impaired Auditory Steady-State Gamma Response. Schizophr Bull. doi:10.1093/schbul/sbx058

Lepock, J. R., Mizrahi, R., Korostil, M., Bagby, R. M., Pang, E. W., \& Kiang, M. (2018). EventRelated Potentials in the Clinical High-Risk (CHR) State for Psychosis: A Systematic Review. Clin EEG Neurosci, 1550059418755212. doi:10.1177/1550059418755212

Light, G. A., Swerdlow, N. R., \& Braff, D. L. (2007). Preattentive sensory processing as indexed by the MMN and P3a brain responses is associated with cognitive and psychosocial functioning in healthy adults. J Cogn Neurosci, 19(10), 1624-1632. doi:10.1162/jocn.2007.19.10.1624

Makransky, G., \& Bilenberg, N. (2014). Psychometric Properties of the Parent and Teacher ADHD Rating Scale (ADHD-RS): Measurement Invariance Across Gender, Age, and Informant. Assessment, 21(6), 694-705. doi:10.1177/1073191114535242 
Miller, T. J., McGlashan, T. H., Rosen, J. L., Cadenhead, K., Ventura, J., McFarlane, W., . . . Woods, S. W. (2003). Prodromal assessment with the structured interview for prodromal syndromes and the scale of prodromal symptoms: predictive validity, interrater reliability, and training to reliability. Schizophr Bull, 29(4), 703-715.

Millier, A., Schmidt, U., Angermeyer, M. C., Chauhan, D., Murthy, V., Toumi, M., \& Cadi-Soussi, N. (2014). Humanistic burden in schizophrenia: a literature review. J Psychiatr Res, 54, 8593. doi:10.1016/j.jpsychires.2014.03.021

Mors, O., Perto, G. P., \& Mortensen, P. B. (2011). The Danish Psychiatric Central Research Register. Scand J Public Health, 39(7 Suppl), 54-57. doi:10.1177/1403494810395825

Näätänen, R. (1990). The role of attention in auditory information processing as revealed by eventrelated potentials and other brain measures of cognitive function. Behavioral and Brain Sciences, 13(02), 201-233. doi:10.1017/s0140525x00078407

O'Donnell, B. F., Hetrick, W. P., Vohs, J. L., Krishnan, G. P., Carroll, C. A., \& Shekhar, A. (2004). Neural synchronization deficits to auditory stimulation in bipolar disorder. Neuroreport, 15(8), 1369-1372.

O'Donnell, B. F., Vohs, J. L., Hetrick, W. P., Carroll, C. A., \& Shekhar, A. (2004). Auditory eventrelated potential abnormalities in bipolar disorder and schizophrenia. Int J Psychophysiol, 53(1), 45-55. doi:10.1016/j.ijpsycho.2004.02.001

Oostenveld, R., Fries, P., Maris, E., \& Schoffelen, J. M. (2011). FieldTrip: Open source software for advanced analysis of MEG, EEG, and invasive electrophysiological data. Comput Intell Neurosci, 2011, 156869. doi:10.1155/2011/156869

Owen, M. J., O'Donovan, M. C., Thapar, A., \& Craddock, N. (2011). Neurodevelopmental hypothesis of schizophrenia. Br J Psychiatry, 198(3), 173-175. doi:10.1192/bjp.bp.110.084384

Polich, J. (2007). Updating P300: an integrative theory of P3a and P3b. Clin Neurophysiol, 118(10), 2128-2148. doi:10.1016/j.clinph.2007.04.019

Psychology Software Tools, I. (2012). E-Prime 2.0. In: Author Sharpsburg, PA.

Purcell, S. M., Wray, N. R., Stone, J. L., Visscher, P. M., O'Donovan, M. C., Sullivan, P. F., \& Sklar, P. (2009). Common polygenic variation contributes to risk of schizophrenia and bipolar disorder. Nature, 460(7256), 748-752. doi:10.1038/nature08185

Rasic, D., Hajek, T., Alda, M., \& Uher, R. (2014). Risk of mental illness in offspring of parents with schizophrenia, bipolar disorder, and major depressive disorder: a meta-analysis of family high-risk studies. Schizophr Bull, 40(1), 28-38. doi:10.1093/schbul/sbt114

Rass, O., Forsyth, J. K., Krishnan, G. P., Hetrick, W. P., Klaunig, M. J., Breier, A., . . Brenner, C. A. (2012). Auditory steady state response in the schizophrenia, first-degree relatives, and schizotypal personality disorder. Schizophr Res, 136(1-3), 143-149.

doi:10.1016/j.schres.2012.01.003 
Rass, O., Krishnan, G., Brenner, C. A., Hetrick, W. P., Merrill, C. C., Shekhar, A., \& O'Donnell, B. F. (2010). Auditory steady state response in bipolar disorder: relation to clinical state, cognitive performance, medication status, and substance disorders. Bipolar Disord, 12(8), 793-803. doi:10.1111/j.1399-5618.2010.00871.x

Reynolds, C. R., Kamphaus, R. W., \& Lutz, F. L. (2003). Reynolds intellectual assessment scales (RIAS). Psychological Assessment Resources.

Ridderinkhof, K. R. (2002). Micro- and macro-adjustments of task set: activation and suppression in conflict tasks. Psychol Res, 66(4), 312-323. doi:10.1007/s00426-002-0104-7

Riva, V., Cantiani, C., Mornati, G., Gallo, M., Villa, L., Mani, E., . . Molteni, M. (2018). Distinct ERP profiles for auditory processing in infants at-risk for autism and language impairment. Sci Rep, 8(1), 715. doi:10.1038/s41598-017-19009-y

Rydkjaer, J., Mollegaard Jepsen, J. R., Pagsberg, A. K., Fagerlund, B., Glenthoj, B. Y., \& Oranje, B. (2017). Mismatch negativity and P3a amplitude in young adolescents with first-episode psychosis: a comparison with ADHD. Psychol Med, 47(2), 377-388. doi:10.1017/S0033291716002518

Schreiber, H., Stolz-Born, G., Kornhuber, H. H., \& Born, J. (1992). Event-related potential correlates of impaired selective attention in children at high risk for schizophrenia Biological Psychiatry, 32(8), 634-651.

Schreiber, H., Stolz, G., Rothmeier, J., Kornhuber, H. H., \& Born, J. (1989). Prolonged Latencies of the N2 and P3 of the Auditory Event-Related Potential in Childrn at Risk for Schizophrenia. European Archives of Psychiatry and Neurological Sciences, 238, 185-188.

Schulze, K. K., Hall, M. H., McDonald, C., Marshall, N., Walshe, M., Murray, R. M., \& Bramon, E. (2008). Auditory P300 in patients with bipolar disorder and their unaffected relatives. Bipolar Disord, 10(3), 377-386. doi:10.1111/j.1399-5618.2007.00527.x

Shaffer, D., Gould, M. S., Brasic, J., Ambrosini, P., Fisher, P., Bird, H., \& Aluwahlia, S. (1983). A children's global assessment scale (CGAS). Archives of General Psychiatry, 40(11), 12281231. doi:10.1001/archpsyc.1983.01790100074010

Shaw, A. D., Knight, L., Freeman, T. C. A., Williams, G. M., Moran, R. J., Friston, K. J., . . Singh, K. D. (2019). Oscillatory, Computational, and Behavioral Evidence for Impaired GABAergic Inhibition in Schizophrenia. Schizophr Bull. doi:10.1093/schbul/sbz066

Sohal, V. S., Zhang, F., Yizhar, O., \& Deisseroth, K. (2009). Parvalbumin neurons and gamma rhythms enhance cortical circuit performance. Nature, 459, 698-702.

Symmonds, M., Moran, C. H., Leite, M. I., Buckley, C., Irani, S. R., Stephan, K. E., . . Moran, R. J. (2018). Ion channels in EEG: isolating channel dysfunction in NMDA receptor antibody encephalitis. Brain, 141(6), 1691-1702. doi:10.1093/brain/awy107

Tada, M., Nagai, T., Kirihara, K., Koike, S., Suga, M., Araki, T., . . Kasai, K. (2016). Differential Alterations of Auditory Gamma Oscillatory Responses Between Pre-Onset High-Risk 
Individuals and First-Episode Schizophrenia. Cereb Cortex, 26(3), 1027-1035. doi:10.1093/cercor/bhu278

Thorup, A. A., Hemager, N., Søndergaard, A., Gregersen, M., Prøsch, A. K., Krantz, M. F., . . . Nordentoft, M. (2018). The Danish High Risk and Resilience Study_VIA 11: Study Protocol for the First Follow-Up of the VIA 7 Cohort -522 Children Born to Parents With Schizophrenia Spectrum Disorders or Bipolar Disorder and Controls Being Re-examined for the First Time at Age 11. Frontiers in Psychiatry, 9. doi:10.3389/fpsyt.2018.00661

Thorup, A. A., Jepsen, J. R., Ellersgaard, D. V., Burton, B. K., Christiani, C. J., Hemager, N., . . Nordentoft, M. (2015). The Danish High Risk and Resilience Study--VIA 7--a cohort study of 520 7-year-old children born of parents diagnosed with either schizophrenia, bipolar disorder or neither of these two mental disorders. BMC Psychiatry, 15, 233-248. doi:10.1186/s12888-015-0616-5

Thune, H., Recasens, M., \& Uhlhaas, P. J. (2016). The 40-Hz Auditory Steady-State Response in Patients With Schizophrenia: A Meta-analysis. JAMA Psychiatry, 73(11), 1145-1153. doi:10.1001/jamapsychiatry.2016.2619

Traub, R. D., Cunningham, M. O., Gloveli, T., LeBeau, F. E. N., Bibbig, A., Buhl, E. H., \& Whittington, M. A. (2003). GABA-Enhanced Collective Behavior in Neuronal Axons Underlies Persistent Gamma Frequency Oscillations. Proceedings of the National Academy of Sciences, 100(19), 11047-11052. doi:10.1073/pnas

Tsai, M. L., Hung, K. L., \& Lu, H. H. (2012). Auditory event-related potentials in children with attention deficit hyperactivity disorder. Pediatr Neonatol, 53(2), 118-124. doi:10.1016/j.pedneo.2012.01.009

Umbricht, D., Koller, R., Schmid, L., Skrabo, A., Grubel, C., Huber, T., \& Stassen, H. (2003). How specific are deficits in mismatch negativity generation to schizophrenia? Biol Psychiatry, 53(12), 1120-1131.

Umbricht, D., \& Krljes, S. (2005). Mismatch negativity in schizophrenia: a meta-analysis. Schizophr Res, 76(1), 1-23. doi:10.1016/j.schres.2004.12.002

Van Voorhis, A. C., Kent, J. S., Kang, S. S., Goghari, V. M., MacDonald, A. W., \& Sponheim, S. R. (2019). Abnormal neural functions associated with motor inhibition deficits in schizophrenia and bipolar disorder. Hum Brain Mapp. doi:10.1002/hbm.24780

Vlaskamp, C., Oranje, B., Madsen, G. F., Mollegaard Jepsen, J. R., Durston, S., Cantio, C., . . . Bilenberg, N. (2017). Auditory processing in autism spectrum disorder: Mismatch negativity deficits. Autism Res. doi:10.1002/aur.1821

Xie, L., Ren, M., Cao, B., \& Li, F. (2017). Distinct brain responses to different inhibitions: Evidence from a modified Flanker Task. Sci Rep, 7(1), 6657. doi:10.1038/s41598-01704907-y 